\title{
A Fatal Case of Venlafaxine Overdose
}

\author{
George M. Bosse, MD ${ }^{a}$, Henry A. Spiller, MS ${ }^{b}$, Aaron M. Collins, MD ${ }^{c}$
}

aDepartment of Emergency Medicine, University of Louisville School of Medicine, Louisville, Kentucky, USA; and Kentucky

Regional Poison Center, Louisville, Kentucky, USA

bKentucky Regional Poison Center, Louisville, Kentucky, USA

${ }^{\mathrm{c} G r e e n v i l l e ~ H o s p i t a l ~ S y s t e m, ~ G r e e n v i l l e, ~ S o u t h ~ C a r o l i n a, ~ U S A ~}$

\begin{abstract}
Introduction: Based on its primary action of serotonin reuptake inhibition, venlafaxine overdose would be expected to result in serotonergic effects.

Case Report: A 40 year old male ingested venlafaxine without co-ingestants in a suicide attempt. The patient developed refractory ventricular fibrillation and expired approximately 9 hours post-ingestion. ECG monitoring revealed significant $\mathrm{QRS}_{\text {and }} \mathrm{QT}_{\mathrm{C}}$ interval prolongation prior to his demise.

Discussion: A literature review of venlafaxine overdose cases and investigation into its mechanism of action was conducted. The potential for sodium channel blockade and implications for therapy are discussed.
\end{abstract}

\section{INTRODUCTION}

Venlafaxine, a bicyclic hydroxycycloalkylphenylethylaminederivative antidepressant, has serotonin, norepinephrine, and dopaminergic reuptake inhibition properties. It is structurally more closely related to tramadol, a non-opioid analgesic, than it is to tricyclic antidepressants (TCA), monoamine oxidase inhibitors (MAOI), or selective serotonin reuptake inhibitors (SSRI) [1,2]. It reportedly does not have the sodium channel blocking properties of TCA, thereby resulting in fewer cardiac side effects [3]. Due to its primary action of serotonin reuptake inhibition, toxic effects after overdose are likely to be similar to those seen with SSRI. It is considered a first-line agent for the treatment of depression [4]. We report a fatal venlafaxine acute overdose without coingestants that produced significant cardiac toxicity.

\section{CASE REPORT}

A 40-year-old male with a history of non-insulin-dependent diabetes mellitus and depression presented to a community emergency department (ED) approximately 45 minutes after intentionally ingesting ninety $150-\mathrm{mg}$ venlafaxine extended release (Effexor XR) and seventy-five 75-mg venlafaxine extended release (Effexor XR) tablets in a suicide attempt. The total amount ingested was $19 \mathrm{~g}$. The medication was prescribed to him for treatment of depression. He had no other medical problems and took no other medications, prescription or over the counter. He denied alcohol, illicit drug, or tobacco abuse.

On initial presentation, the patient was asymptomatic except for nausea. Initial vital signs were heart rate $136 \mathrm{bpm}$, blood pressure $133 / 90 \mathrm{mmHg}$, respiratory rate $16 / \mathrm{min}$, pulse oximetry $99 \%$ on room air, and weight $106.8 \mathrm{~kg}$. No temperature was recorded. Physical examination revealed an alert male in no distress with clear lungs, a soft abdomen, and a non-focal neurologic examination. Cardiac exam revealed tachycardia with a regular rhythm. The patient received a $500 \mathrm{~mL}$ bolus of normal saline. Fifty grams of activated charcoal with sorbitol was administered orally. Acting on recommendations from the regional poison control center, whole-bowel irrigation was initiated with polyethylene glycol solution at $1-2 \mathrm{~L} / \mathrm{hr}$. The initial electrocardiogram (ECG) revealed

Keywords: venlafaxine; fatality; sodium channel poisoning; case report

Notes: There was no outside funding of any kind used for this study.

Corresponding Author: George M. Bosse, Department of Emergency Medicine C1H17, University of Louisville Hospital, 530 S. Jackson St, Louisville, KY 40202 
Table 1: ECG characteristics from time of ingestion

\begin{tabular}{lccc}
\hline Time from ingestion & PR interval (msec) & QRS interval (msec) & QTc (msec) \\
\hline $1 \mathrm{hr}, 35 \mathrm{~min}$ & 143 & 90 & 422 \\
\hline $4 \mathrm{hr}, 20 \mathrm{~min}$ & 144 & 112 & 425 \\
\hline $8 \mathrm{hr}, 40 \mathrm{~min}$ & 400 & 158 & 564 \\
\hline$E C G=$ electrocardiogram, $\mathrm{hr}=$ hours, min $=$ minutes, msec = milliseconds. \\
\hline
\end{tabular}

sinus tachycardia with no ischemic changes (Table 1). The comprehensive metabolic profile and complete blood count were normal. Acetaminophen, salicylate, and ethanol levels were undetectable. The patient had two episodes of emesis. He was noted to be sleepy but arousable approximately two hours postingestion, at which time he was transferred to a tertiary care facility. During transport the patient had a tonic-clonic seizure lasting 2-3 minutes.

The patient arrived at the tertiary ED 3.5 hours post-ingestion. His presenting vital signs at the referral ED were heart rate 140 bpm, blood pressure $116 / 65 \mathrm{mmHg}$, respiratory rate $38 / \mathrm{min}$, temperature $37.5^{\circ} \mathrm{C}$, and pulse oximetry $96 \%$ on room air. He was described as alert, oriented, in no acute distress, and diaphoretic. No other abnormalities were identified on physical exam. He subsequently had a third episode of emesis. He was given $25 \mathrm{mg}$ intravenous (IV) promethazine for continued nausea. A second tonic-clonic seizure was observed by the ED staff 4.5 hours postingestion, for which he was given $2 \mathrm{mg}$ IV lorazepam.

Urine drug screen was positive for phencyclidine, and negative for amphetamines, barbiturates, benzodiazepines, cannabinoids, cocaine, and opiates. Confirmational testing of the phencyclidine was negative. A repeat ECG showed sinus tachycardia (see Table 1), and his chest radiograph was normal. He was admitted to the intensive care unit for further monitoring and supportive treatment. He became progressively more lethargic, and approximately 9 hours post-ingestion developed refractory ventricular fibrillation (VF) and subsequently expired. An ECG, showing no ventricular ectopy, was obtained just prior to his demise (see Table 1).

A venlafaxine blood level obtained 5 hours post-ingestion was $9000 \mathrm{ng} / \mathrm{mL}$, and the 0-desmethylvenlafaxine blood level, the first metabolite of venlafaxine, was $3000 \mathrm{ng} / \mathrm{mL}$. A postmortem venlafaxine blood level drawn from a peripheral site was $5500 \mathrm{ng} / \mathrm{mL}$. No other substances were found in the post-mortem analysis.

\section{DISCUSSION}

The patient we present had a clear progression of his QRS and QTc prolongation with subsequent refractory ventricular fibrillation, suggesting that sodium channel poisoning may have been responsible for his dysrhythmia and subsequent death. This patient had no signs of serotonin syndrome such as rigidity, clonus, tremor, or hyperthermia.
This patient had a positive urine immunoassay for phencyclidine with negative confirmational testing. Although this patient denied taking any other medication, a potential coingestant such as tramadol or diphenhydramine could potentially explain the positive test. However, studies have shown that venlafaxine alone can cause false-positive urine immunoassay results for phencyclidine $[5,6]$.

This patient also received one 25-mg dose of promethazine. Promethazine has been shown in guinea pig myocytes to have similar sodium channel blocking effects as class I antidysrythmics [7]. Despite this action, it is unlikely with one 25-mg dose to have contributed significantly to the patient's clinical course.

This case is illustrative due to the clear documentation and observation of the patient's symptoms, cardiac manifestations, and clinical decline from very early on in his ingestion through the terminal event. There was a relatively rapid progression of his cardiac dysrhythmia and atrioventricular block.

Venlafaxine is an atypical antidepressant whose actions primarily involve inhibition of serotonin reuptake in presynaptic neurons. At therapeutic doses, it has minor effects on the reuptake of norepinephrine and dopamine, which is probably not clinically relevant [1]. Venlafaxine overdose has been reported to cause seizures, dysrythmias, central nervous system (CNS) depression, and hypotension [3,8].

In a case series of SSRI, venlafaxine, and TCA overdoses, venlafaxine-induced QRS prolongation occurred more frequently than TCA-induced QRS prolongation [1]. The study also compared the rates of seizures, serotonin toxicity, and ICU admissions between SSRI, venlafaxine, and TCA. Seizures, as well as serotonin syndrome, occurred more frequently with venlafaxine overdose [1]. The incidence of ICU admission is greater for venlafaxine than SSRI, though less than that of TCA [1].

Previous reports suggest that venlafaxine does not have the sodium channel toxicity manifested by the TCA [3]. However, one potential mechanism for the cardiac toxicity observed with venlafaxine may relate to sodium channel poisoning. In a guinea pig myocyte model, venlafaxine significantly reduced the sodium channel conduction rate, specifically by acting on the fast inward sodium current, the primary regulator of cardiac depolarization [9]. This effect was dose dependent and conduction returned to normal with removal of the venlafaxine. These experiments were done with a venlafaxine concentration of $10^{-6} \mathrm{M}$. Steady-state therapeutic concentrations in humans have been reported to range from $2 \times 10^{-7}-10^{-6} \mathrm{M}$, with overdose concentrations in 
humans easily exceeding $3 \times 10^{-5} \mathrm{M}(10-12)$. The sodium channel poisoning effect of venlafaxine may occur with binding to the resting state of the channel, which differs from the effects of TCA and traditional class I antiarrhythmic drugs [9].

Other pharmacokinetic processes may also contribute to venlafaxine's toxicity, including significant differences in the metabolism of venlafaxine between individuals with extensive vs. poor metabolizer CYP2D6 phenotypes [4]. Patients who may be poor CYP2D6 metabolizers, or patients receiving medications known to inhibit CYP2D6 may be at greater risk for toxicity from venlafaxine [4]. In addition, venlafaxine overdose resulting in serotonin syndrome, a potentially fatal reaction, has been reported [13].

Therapy for venlafaxine overdose has not been well established. The impact of early supportive treatments-including intubation and fluid resuscitation-are unknown in this setting, but should be utilized in any unstable patient. This patient's initial tachycardia may represent effects from beta receptor stimulation from excess dopamine and norepinephrine from toxic levels of venlafaxine; the significance and prognostic value of this is one area for future research. Given the possibility of sodium channel poisoning, sodium bicarbonate therapy in venlafaxine overdose associated with widened QRS on ECG or ventricular dysrhythmias may be beneficial, and further investigation of its use may prove valuable. Sodium bicarbonate is used in similar settings of cardiac toxicity secondary to TCA and related agents.

Until further research clarifies these questions, it may be prudent to recommend prolonged observation in patients presenting with initial tachycardia, and to suggest administration of sodium bicarbonate at the first signs of QRS widening.

The authors have no potential financial conflicts of interest to report.

\section{REFERENCES}

1. Whyte IM, Dawson AH, Buckley NA. Relative toxicity of venlafaxine and selective serotonin reuptake inhibitors in overdose compared to tricyclic antidepressants. Q J Med 2003;96: 369-374.
2. Jaffe PD, Batziris HP, van der Hoeven P, DeSilva D, McIntyre IM. A study involving venlafaxine overdoses: comparison of fatal and therapeutic concentrations in postmortem specimens. J Forensic Sci 1999;44(1):193-196.

3. Peano C, Leikin JB, Hanashiro PK. Seizures, ventricular tachycardia, and rhabdomyolysis as a result of ingestion of venlafaxine and lamotrigine. Ann Emerg Med 1997;30(5):704-708.

4. Lessard E, Yessine MA, Hamelin BA, O'Hara G, LeBlanc J, Turgeon J. Influence of CYP2D6 activity on the disposition and cardiovascular toxicity of the antidepressant agent venlafaxine in humans. Pharmacogenetics 1999;9:435-443.

5. Bond GR, Steele PE, Uges DR. Massive venlafaxine overdoses resulted in a false positive Abbott AxSYM urine immunoassay for phencyclidine. J Tox Clin Tox

2003;41(7):999-1002.

6. Sena SF, Kazimi S, Wu AH. False-positive phencyclidine immunoassay results caused by venlafaxine and O-desmethylvenlafaxine. Clin Chem 2002;48(4):676-677.

7. Tanaka H, Habuchi Y, Nishimura M, Sato N, Watanabe Y. Blockade of $\mathrm{Na}^{+}$current by promethazine in guinea-pig ventricular myocytes. Br J Pharmacol 1992;106:900-905.

8. Blythe D, Hackett LP. Cardiovascular and neurological toxicity of venlafaxine. Hum Exp Tox 1999;18:309-313.

9. Khalifa M, Daleau P, Turgeon J. Mechanism of sodium channel block by venlafaxine in guinea pig ventricular myocytes. J Pharmacol Exp Ther 1999;29(1):280-284.

10. Klamerus KJ, Maloney K, Rudolph RL, Sisenwine SF, Jusko WJ, Chiang ST. Introduction of a composite parameter to the pharmacokinetics of venlafaxine and its active O-desmethyl metabolite. J Clin Pharmacol 1992;32:716-724.

11. Patat A, Troy S, Burke J, Trocherie S, Danjou P, Le Coz F, Allain H, Gandon JM. Absolute bioavailability and electroencephalographic effects of conventional and extended-release formulations of venlafaxine in healthy subjects. J Clin Pharmacol 1998;38:256-267.

12. Kokan L, Dart RC. Life-threatening hypotension from venlafaxine overdose (Abstract). Ann Emerg Med 1996;27:815.

13. Kolecki P. Isolated venlafaxine-induced serotonin syndrome. J Emerg Med 1997;15(4):491-493. 